\title{
Conception and Implementation of an IoT System for Remote Practical Works in Open Access University's Electronic Laboratories
}

\author{
https://doi.org/10.3991/ijoe.v17i02.19755 \\ Amine Moulay Taj $(\bowtie)$ \\ Sultan Moulay Slimane University of Beni Mellal, Beni Mellal, Morocco \\ Mytaj.amine@gmail.com \\ Jesus Chacon Sombria \\ Universidad Complutense de Madrid, Madrid, Spain \\ Ahmed Gaga, Abdelmoula Abouhilal, Abdessamad Malaoui \\ Sultan Moulay Slimane University of Beni Mellal, Beni Mellal, Morocco
}

\begin{abstract}
The courses and disciplines in the exact sciences are based on practical work (PW) which complements the theoretical teaching. Remote Labs are a recent approach used for educating experimental manipulations by using the performance of information and communication technologies. This work aims to propose the design of an IoT system allowing several remote manipulations in analog electronics. This solution is mainly based on the Red Pitaya STEMLab board, in particular for communication and remote control for measurements and data acquisition through different protocols. The low-cost system provides an intelligent selection between the different integrated practical works on a developed board, as well as other ones carried out externally as extensions according to the professor's needs. The analysis of the integrated manipulations was done taking into account the minimum latency of the remote control as well as its portability to save time, space, and money without loss of quality and quantity. The presented results relate to a PW of analog electronics, a "RC charging and discharging circuit" has been considered for a comparison of this Remote system with the same PW performed by the classical method "hands-on laboratory". Our purpose in this study is the development of a control system to maximize the available resource as well as to improve self-learning ability in the electronic field.
\end{abstract}

Keywords - Open-source hardware, remote laboratories, engineering education, educational system design, smart educational board

\section{Introduction}

In a developing country, economic, social and cultural progress can only be achieved through higher education that produces quality skills. Consequently, this pillar of the country's development helps to address social issues and challenges it faces in the 
context of internationalization [1-4]. It trains highly qualified youth capable of taking charge of enterprises in different sectors of the economy and public service organizations. Hands-on sessions are an essential part of university curricula, they offer another entry into the subject, another rhythm, with a smaller group of students [5-6]. These activities have proved to be essential in scientific and technical training tasks in which students manipulate real objects or materials or witness a demonstration by the teacher, particularly in technical streams such as engineering, where students are required to complete practical exercises and assignments essential to their learning [7].

Unfortunately, the overcrowding of higher education institutions in developing countries is one of the main problems of theoretical education, which takes precedence over practical education in a more remarkable way. In this situation, it is not only students who deplore this situation, teachers are also very dissatisfied with this overcrowding of universities. There are too many students for the same infrastructure, the same equipment, but also the same teacher [8].

In the Electronic section, students have their first contact with resistors and capacitors, as well as with the oscilloscope and the signal generator, in the first analog electronics courses, especially during practical work. Students are divided into small groups and assigned to a limited number of manipulations and supervised by a teacher, They learn how to make the first resistance assemblies, how to analyze their different combinations (series, parallel), and how to see how a voltage divider works. They also do their first practical exercises with diodes in their rectifier configuration and see slightly more complex circuits such as filters or voltage limiters based on Zener diodes. However, to perform these practices, it is also necessary to use the laboratory equipment: oscilloscope, signal generator, multimeter, and power supply.

The subject is complex when you add to that the teacher's warnings on the use of the equipment, the fear of working with high voltages, and the fact that this is the first time the students are in a laboratory where not only they have to understand and assemble the circuits but also understand and see how the equipment works with the change of each component value in the practical work, this configuration which is done manually by the students takes time and involves risks in parallel.

Thanks to the development of Internet technologies and new methods of information sharing, which have facilitated the emergence of various e-learning scenarios. The best known in the practical pillar of education is the creation of remote-controlled laboratories [9-11]. In a remote laboratory, the same interaction between students and real equipment takes place remotely with the help of infrastructure based on client-server architecture [12]. The latter provides a new layer between the user and the laboratory equipment, it is in charge of transmitting user actions and receiving sensory information from the equipment. In parallel with open-source hardware movement, which is becoming increasingly popular due to the emergence of low-cost, high-performance technologies such as Arduino and Raspberry Pi, has contributed significantly to the development of this type of remote-controlled laboratory [13]. Although the interest in this new concept is growing, the actual service offer is still very limited.

The objective of this paper is to propose the conception and design of an IoT System for remote practical works in the university's electronic laboratories. This solution is mainly based on the Red Pitaya card, in particular for communication and remote 
control by RIP protocol, for measurements and data acquisition, to which is added an electronic board allowing an intelligent selection between the different integrated practical works as well as other manipulations carried out outside as extensions according to the professor's needs. The developed board supports different control protocols namely SPI, UART, I2C, as well as it allows to control the value of the resistances in all the manipulations in a continuous way. The analysis of the manipulations has been made taking into account the minimal latency of the remote control as well as its portability to save time, space, and money without loss of quality and quantity.

The remainder of this paper is organized as follows. Section 2 covers related work. Section 3 introduces the general architecture of the IoT system. Section 4 deals with the experimental and Implementation of the IoT system. Section 5 reports our evaluation results. Finally, Section 6 provides some concluding remarks.

\section{Related Work}

In recent years, there are several methods to approach electronic engineering, the majority of them focusing on motivating students to learn [14-15]. Videos demonstrating phenomena, as well as free and web-based interactive simulators, have been applied in teaching, with fruitful and promising results [16].

The use of practical kits and platforms on which pedagogical experiments are carried out has continued to evolve [17-18]. There is generally a strong demand in the industry for the skills acquired from these educational platforms [19]. Among others, Practical projects reinforce the concepts presented in class and provide greater insight into various design issues that may not be evident in theory.

On the other hand, new strategies are being used to engage and motivate students to learn electronics, which means that teaching and learning about electronics cannot be limited to methods that focus on the traditional classroom environment. Due to the emergence of low-cost high-performance technologies such as Arduino and Raspberry $\mathrm{Pi}$, the open-source hardware movement is growing in popularity, thanks to the community of manufacturers who actively share their creations to be studied, modified, and redistributed freely [13].

Remote laboratories are a new tool to complement in-person laboratories, simulators, and virtual laboratories. The pool resulting from all the available possibilities provides a wide range of possibilities when designing a course in which experimentation plays a key role. Various researchers have examined the suitability of remote and virtual laboratories for analog electronics [20-21] and recommended that remote laboratories should not be used in this area. After some years of the technical evolution of remote laboratories, this assertion was challenged [22-23] by researchers who established the suitability of analog electronics remote laboratories from a methodological point of view.

Among the popular remote laboratory dedicated to electronics we can mention the following: 
- VISIR, (Virtual Instrument Systems In Reality) [24], which is a Remote Laboratory developed in the Blekinge Institute of Technology, which supports remote experimentation with real electronic circuits. The VISIR remote laboratory was created in the late 1990s as a research project at the Blekinge Institute of Technology, Sweden. VISIR is the world's most powerful and most popular electronics project (and received the "Best Online Laboratory Award" from the GOLC and the Global Online Laboratory Consortium in 2015).

- NetLab [25], which is an interactive learning environment that also allows students far away from each other to collaborate and as such, it is a unique system. NetLab is a distance laboratory-developed at the University of South Australia and has been used in teaching students for over 15 years. The development of NetLab began in 2001 and was funded by a Teaching and Learning Grant from the University of South Australia. The system has been continually improved over time by including more features to enhance the learning experience for students, but also by providing academic staff with information on how students use the system.

- ISILab, (Internet Shared Instrumentation Laboratory) [26], developed at the University of Genoa, is currently used to deliver online access to experiments on electronics for the benefit of a few engineering courses. It allows practicing with electronic instruments and measurement methods, executing real experiments of scalable complexity on analogical and digital circuits. The experiments deal with basic electronic measurements, such as delays in digital circuits or the gain and the distortion of amplifiers, and use devices such as waveform generators and oscilloscopes.

- RemotElecLab [27] which is a newer remote laboratory platform for experimentation with electrical and electronic circuits. It has been developed after a study of the disadvantages of existing remote laboratory solutions for the same type of experiments, to bypass them, for example by using generic equipment and it is accessed through a generic interface that does not depend on the circuit being tested.

\section{Architecture of the IoT System}

The following section describes the organization of the different elements that generate our system of remote practical work in electronics. It is a client-server architecture based on the combination of two subsystems, the STEMLab Red Pitaya card and the smart board that we have developed, especially for the communication and remote control of measurements and data acquisition on the card developed through different protocols. 
University Compus / Fablab

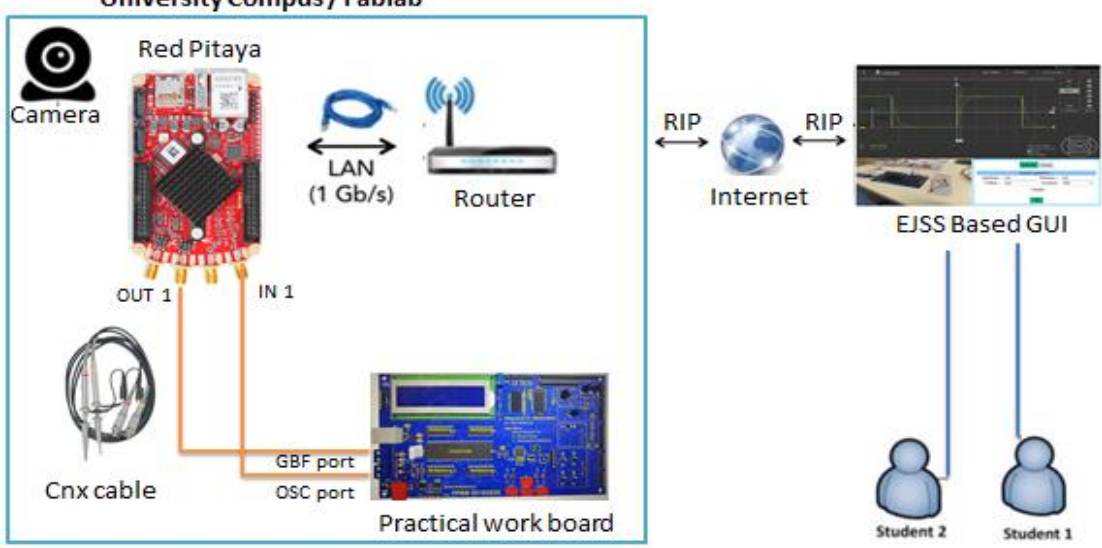

Fig. 1. A general architecture for the remote IoT system for practical work

In this work, the Remote Interoperability Protocol (RIP) [28], which was developed at UNED for the remote operation of online labs, was used to ensure the translation of the student's command into the local installation and to visualize the response of the controlled circuit on the redPitaya interface screen. The architecture allows the control of the Red Pitaya, which in turn controls the manipulation on the developed board.

Indeed, Red Pitaya is an open-source IT project designed to replace many expensive laboratory measurement and control instruments. The card has a graphical interface to simulate a generator, an oscilloscope, and many other devices that are essential for practical work in electronics. It is part of the open-source hardware (OSHW) family, using readily available components and materials, standard processes, open and unrestricted infrastructure, and open-source design tools to maximize the content and quality of information as well as people's ability to build and use computer equipment[29]. To access the Red Pitaya interface, simply connect the card to the same network as a computer and then enter the card's IP address into the browser, Figure 2 shows the composition of the Red Pitaya card.

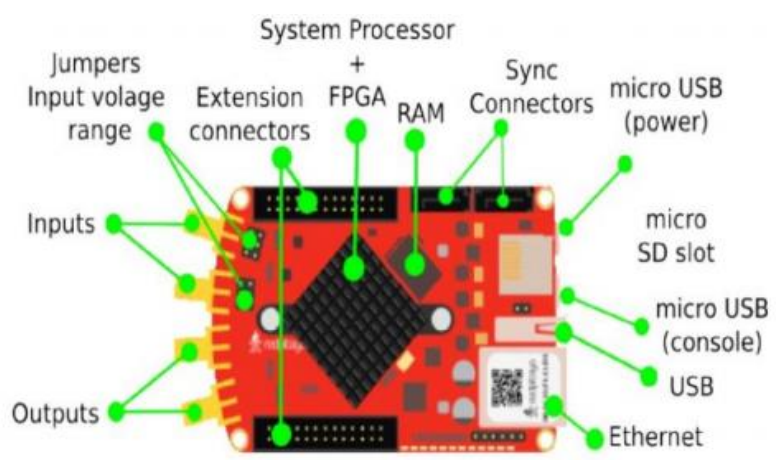

Fig. 2. The components of the Red Pitaya card 
The developed board allows intelligent selection between the different integrated practical work as well as for other manipulations carried out externally as extensions according to the needs of the teacher. The students have access to a web interface that allows them to select a practical work, which presents an interactive electronic diagram and a description of the practical work and the tasks required to complete the practical work.

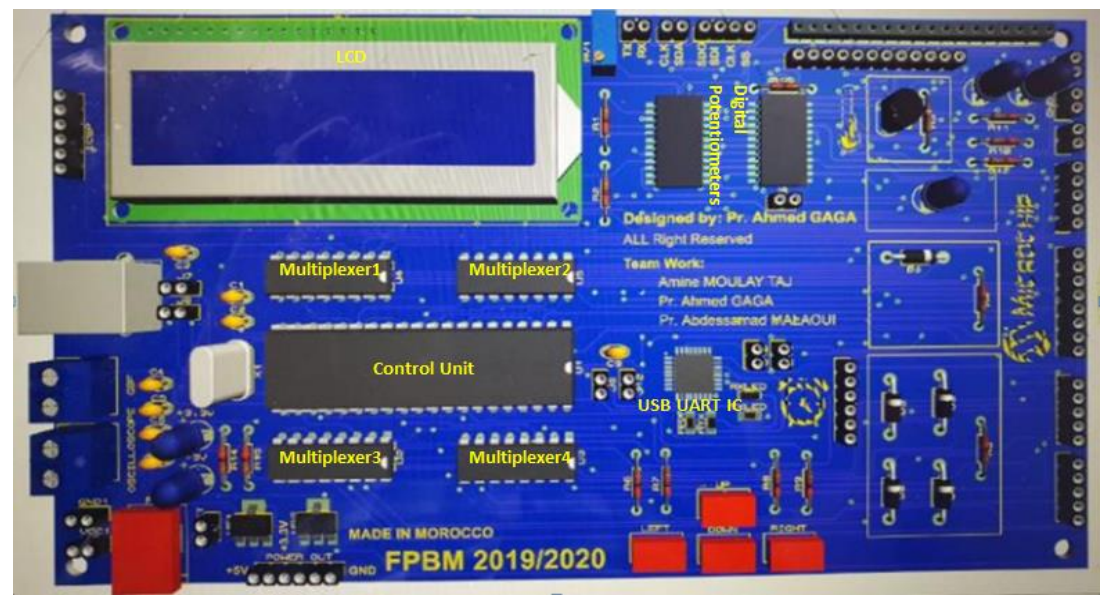

Fig. 3. A simulation of the developed board

The architecture of our work is based on the Red Pitaya card mainly because it can be controlled remotely via a LAN or wireless interface using Matlab, LabVIEW, Scilab, or Python. The Red Pitaya interface is commonly used to control test and measurement instruments for development.

\section{Experimental and Implementation of the IoT System}

This part focuses on the structure of the developed board and the added value it brings compared to the standard method of remote control of the electronics manipulations, detailed description of the integrated components as well as the design adopt-ed is presented.

Learners generally need time to become familiar with the equipment and overcome these fears of manipulating buttons, spinning wheels, and connecting cables. But unfortunately, time is short, because every week there are new practices and it is always necessary to have assimilated the above. Besides, the labs are often busy with classes and many students need extra time outside of class hours to do the practices. In this scenario, a remote lab can help manage and resolve the tensions inherent in practicing from home.

When designing a practical remote work in electronics according to a client-server architecture. The standard way is to translate the electronic schematic of the work on a test plate in which all the components containing the electronic schematic are placed 
and connected with wires as shown in Figure 3. at this stage, two problems related to size and configuration are posed.

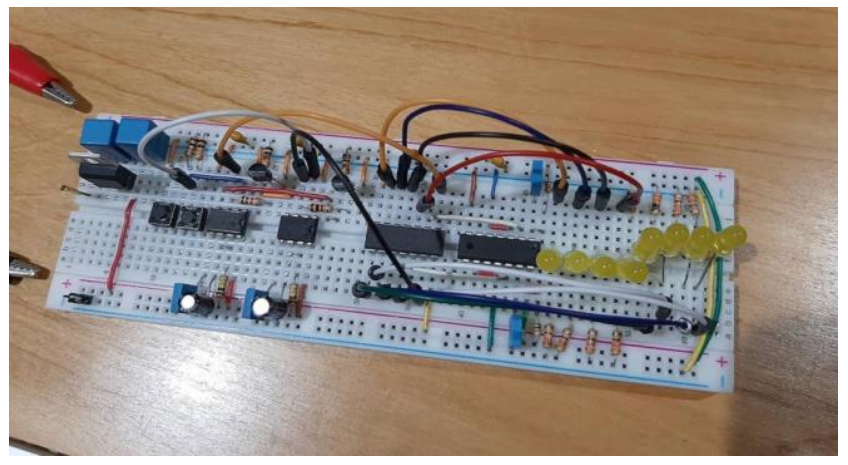

Fig. 4. Example of practical work on the breadboard in electronics

In order to allow the students to change the values of the components as well as the inputs of the signal generated by a generator and the outputs displayed on the oscilloscope. The tutor must set different values for each component and use a large number of links that will be controlled by the student interface to configure the electronic schematic to work with the defined values of the components to deduce the impact of these changes on the output signal of the oscilloscope.

For example, in the case of studying the transient response of the RC circuit, the value of the resistor and capacitor in the circuit should be multiple, for the resistor a series of values such as (10 ohms, $20 \mathrm{ohms}, 50 \mathrm{ohms})$. The effort of the teacher who prepares the practical design of the work is not negligible in this situation.

We propose as a solution an electronic board that gathers six practical tasks in a single small plate with fluidity in the way of controlling the electronic parameters and inputs/outputs of each manipulation. This solution allows for answering the challenge of electronic remote control without using relays.

The integrated manipulations are the:

- Transient Response of RC Circuit

- Transient Response of an RL Circuit

- Voltage Division

- Single-ended rectifier

- Inverting amplifier

- Full-wave rectifier 


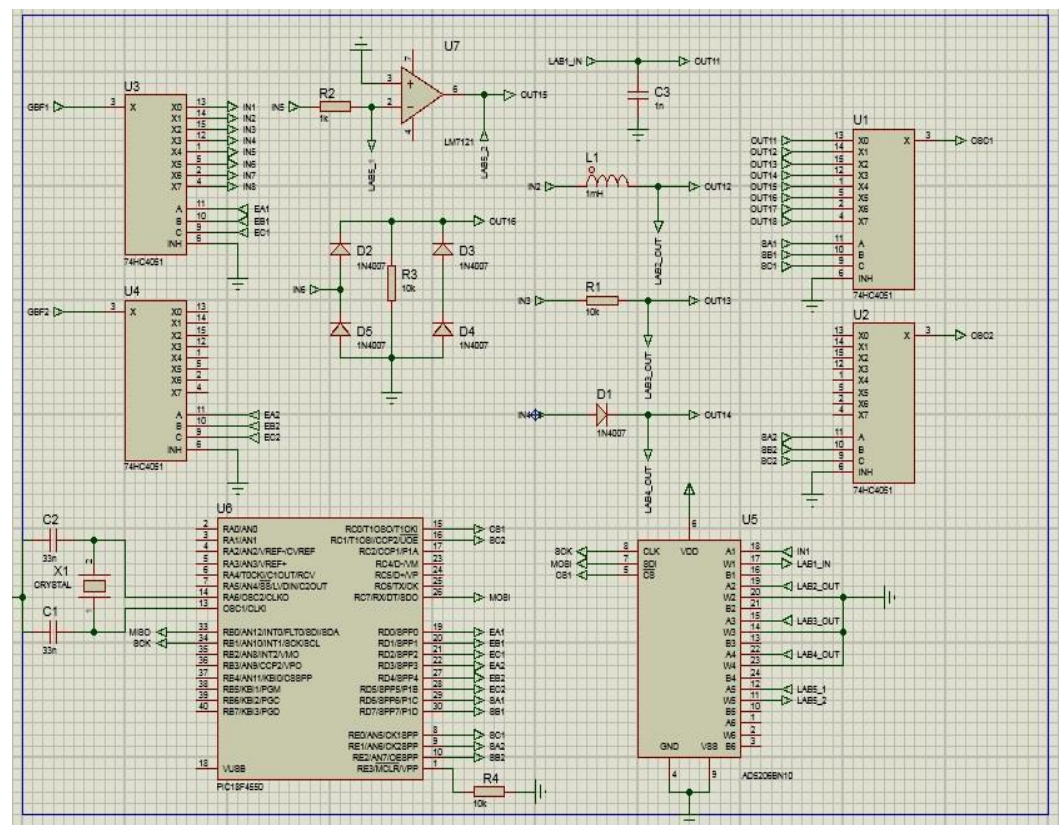

Fig. 5. Electronic schematic of the proposed board

An analog multiplexing technique to route input/output signals to and from the selected manipulations has been integrated. It supports two inputs and outputs signals, increasing the number of possibilities offered by the system.

The selection is made by a control system which is a kind of microcontroller responsible for two main tasks:

- The selection of the desired manipulation among the six integrated manipulations and for $\mathrm{N}$ possibilities created by the teacher outside the board.

- The control of the values of the passive elements used (Resistance) via the SPI protocol (Serial Peripheral Interface).

- The proposed board integrates two digital potentiometers that are controlled via the SPI (Serial Peripheral Interface) protocol to control the resistance value continuously, the first one is connected to the six integrated manipulations, and the second one is dedicated to modifying the resistance value in the extensions created by the teacher outside the board. This property is an added value to the quality of the board. 


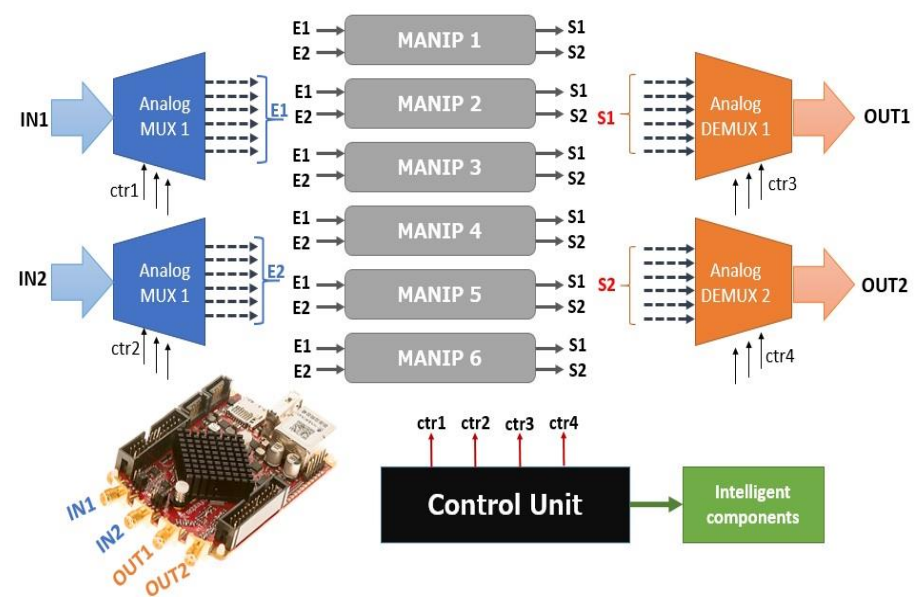

Fig. 6. Synopsis illustrating the multiplexing technique adopted on the proposed board

The Red Pitaya card is used in architecture as a mini-computer that can be remotely controlled via a web interface in the Internet network. Students can control the input signal, this signal passes through an analog multiplexer which is in charge of its distribution in all manipulations, Then students can choose the desired manipulation and change the values of the parameters of each circuit, then visualize the output signal in the same interface. The following figure shows the final design of the developed board.

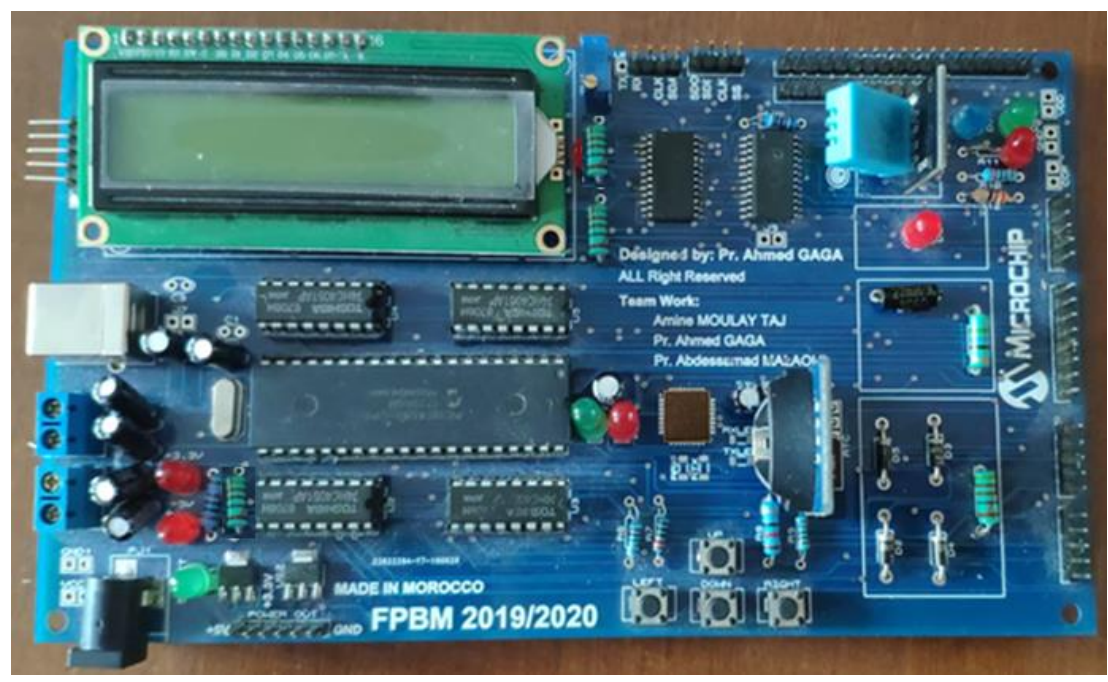

Fig. 7. The real developed board 


\section{$5 \quad$ Results and Discussion}

The results of this work are presented in two steps. The first stage concerns the enduser interface and the technology used to perform the remote practical work. The second step concerns the evaluation of the IoT system, a practical work-related to analog electronics have been considered for the remote laboratory experiment. The "RC charge and discharge circuit" remote practical work is used for a comparison with the same practical manipulations that are performed in the classical system under the same conditions.

\subsection{The user interface}

The overriding importance of creating remote labs focused on creating an easy and flexible environment as possible for students at all times. The technique described in this article aims to reduce financial and human resources as well as improve the capacity for self-directed e-learning in the field of electronics. This part deals with the description of the technology and the communication protocol implemented in the different parts of our system in order to realize the end-user interface as a final result shown in Figure 8.

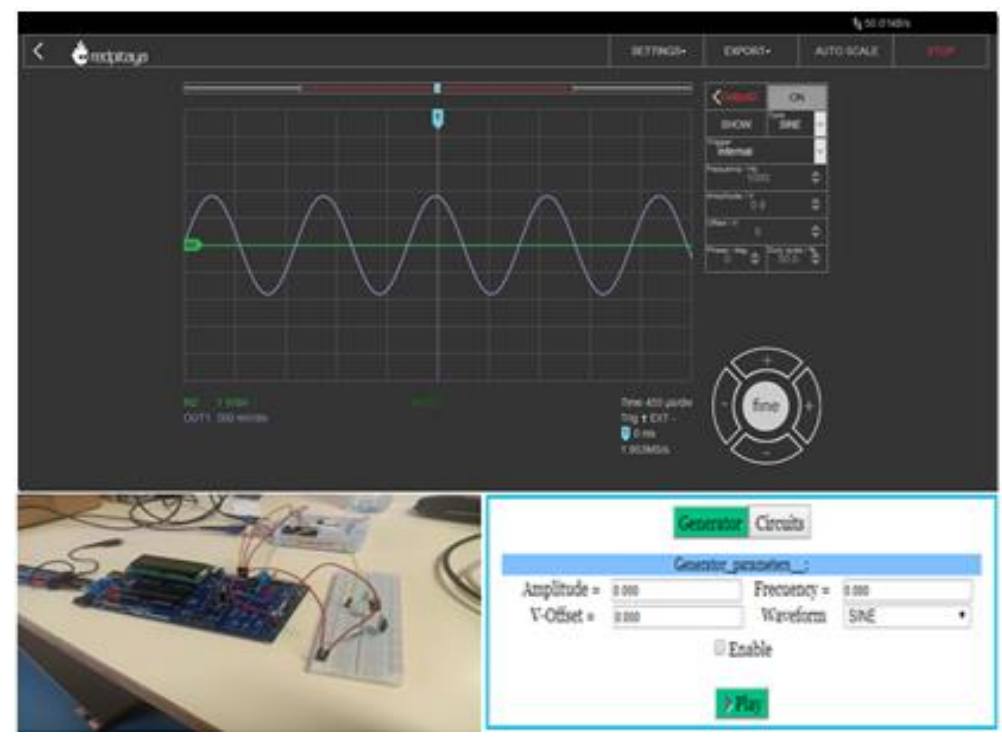

Fig. 8. The control interface developed using EJSs

The generator and the oscilloscope are quite sophisticated and expensive measuring devices; they are indispensable tools when you are doing electronics. The IoT system we developed is based on the red pitaya card which is an open-source IT project designed to replace many expensive laboratory measurement and control instruments, namely Generator and oscilloscope. An interface web was developed for configuring 
the generator inside the Red Pitaya card also to see the circuit response on the oscilloscope on the same interface, EJSS (Easy Java Simulation), which is a free authoring tool written in Java that allows the creating of interactive simulations in Java or Javascript, mainly for teaching and learning purposes, has been used to develop our interface [30].

The communication between the Red Pitaya card and the developed interface is based on the Remote Interoperability Protocol (RIP), which was designed at UNED for the remote operation of online laboratories [28]. It enables the use of Arduino, MATLAB, Red Pitaya (and many more) programs through the Internet as web services. The RIP protocol is used by RIP client and RIP server to communicate both. The RIP server is implemented in the Red Pitaya, and the RIP client is implemented in the EJSS interface.

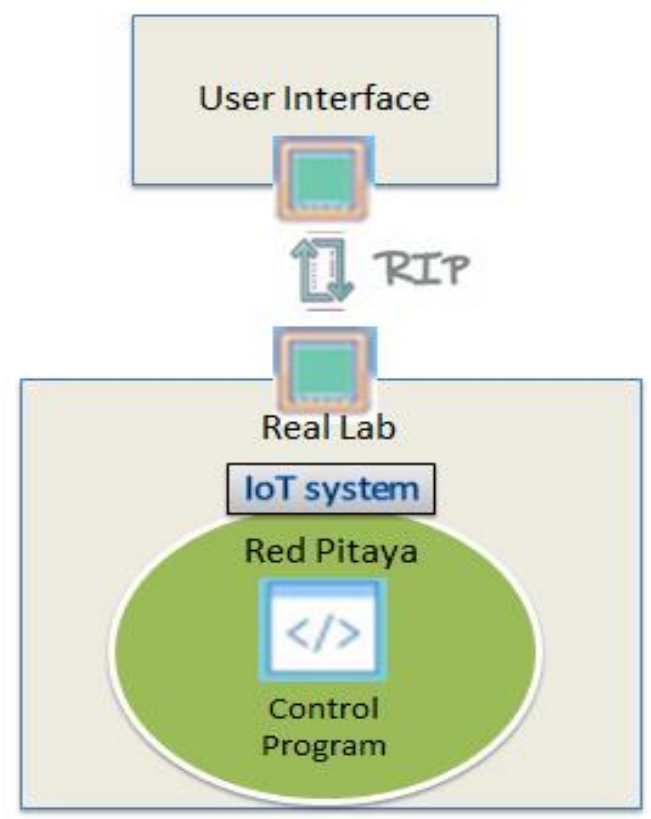

Fig. 9. Illustration of the communication between The user interface and the IoT system

The RIP python server with a control program was implemented on the Red Pitaya card, the control program in charge of controlling the Red Pitaya generator is presented in Figure10. The configuration for allocating the communication between the developed interface and the Red Pitaya card is described in the following link: https://github.com/MyTaj-Amine/rip-python-server/blob/master/config-examples/ AppConfig-RedPitaya.py 


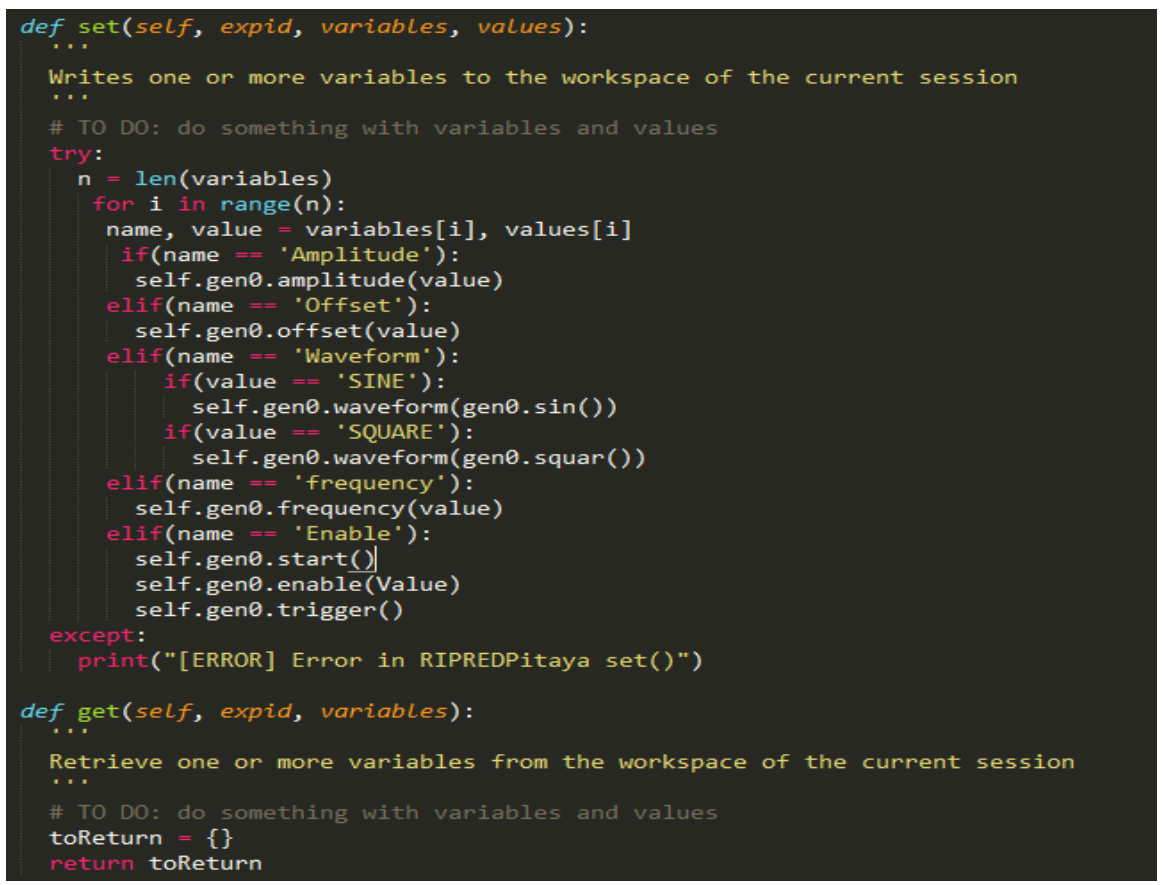

Fig. 10. Part of the control program responsible for controlling the red pitaya generator

\subsection{Evaluation of the IoT system}

Capturing students' perceptions of their learning experiences in different dimensions is an important issue in the evaluation process of any practical work system. Among the practical tasks integrated into the developed system, the RC charge and discharge circuit was considered for a remote laboratory experiment, which is compared to the same practical tasks performed in the laboratory. Figure 11 illustrates the remote experiment of charging and discharging of a capacitor.

The main objectives of this laboratory experiment are described below:

1. To describe the variation of charge versus time for both charging and discharging Capacitor

2. To derive the relationship between the charge stored in a capacitor and the voltage across its plates

3. To calculate the capacitance from the measured voltage and time calculated at the half-time voltage 


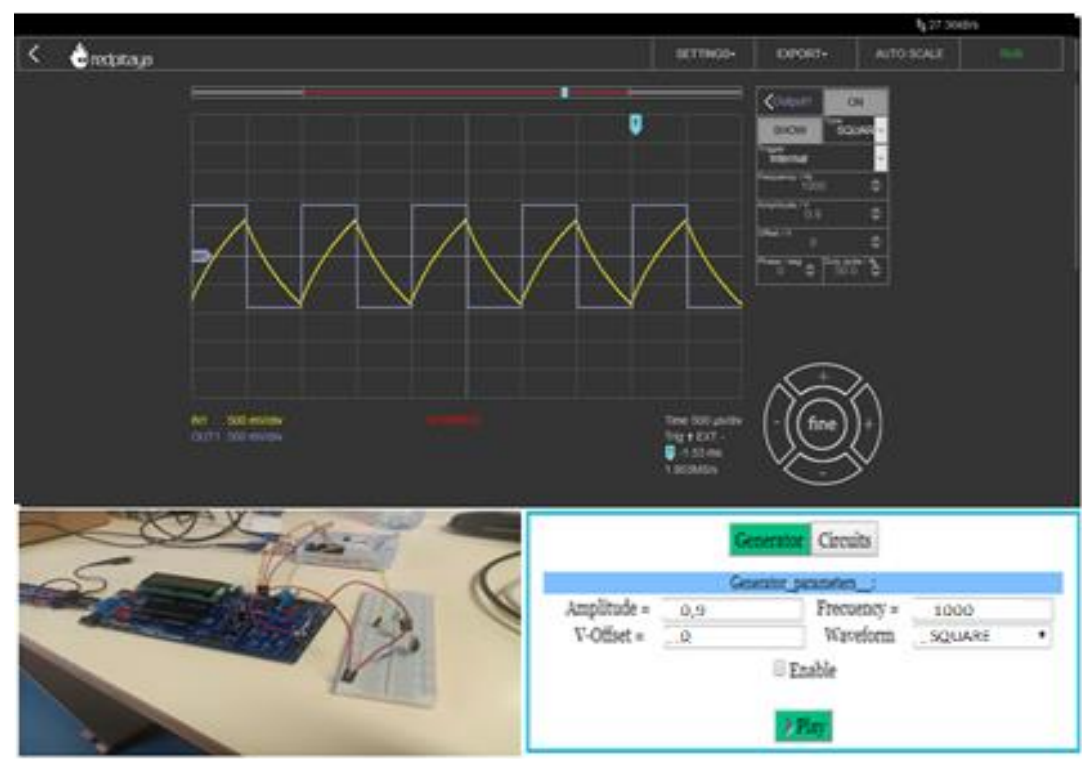

Fig. 11. Charging and discharging of a capacitor using the remote IoT system

The evaluation of the IoT system was conducted among 24 students enrolled in the 3rd year of the "electronic" course at the Sultan Moulay Slimane University (Beni Mellal - Morocco). They were divided into two groups; the first one handles the manipulation locally, where the second use the remote IoT system for PW. The authors adopt the same questionnaire and the same factor applied in the same university to validate a new strategy of online practical work on power electronics for embedded systems in 2017 [6]. A K factor is calculated using both equations (1) and (2), where $S$ is the average of the student responses for each question and $\mathrm{K}$ is the percentage of $\mathrm{S}$ by the number of choices in each question. In our case $\mathrm{N}=12$ represents the number of students in each group, $M=4$ is the number of answers for each question and The " $R_{j}$ " is the response of student $\mathrm{j}$ for each question. The questionnaire contains four closed questions ( $\mathrm{Q} 1$, Q2, Q3, and Q4) and two open questions (Q5 and Q6), see tables 1.

$$
\begin{aligned}
& \mathrm{S}=\frac{1}{N}+\sum_{j} R_{J} \\
& \mathrm{~K}=\frac{S}{M} * 100 \%
\end{aligned}
$$


Table 1. Evaluation questionnaire

\begin{tabular}{|c|c|c|c|c|}
\hline \multicolumn{5}{|c|}{ Closed questions } \\
\hline Factor & Questions & Response & Hands-on PW (\%) & Remote PW (\%) \\
\hline K1 & $\begin{array}{l}\text { Q1: How is difficult is to have access } \\
\text { to all the different PW elements? }\end{array}$ & $\begin{array}{l}\text { 1-Very difficult } \\
\text { 2- Quite difficult } \\
\text { 3- minor difficul- } \\
\text { ties } \\
4-\text { No problem }\end{array}$ & 98.5 & 84.3 \\
\hline K2 & $\begin{array}{l}\text { Q2: Are the PW documents clear } \\
\text { enough about what you have to do? }\end{array}$ & $\begin{array}{l}\text { 1-Very clear } \\
\text { 2- Quite clear } \\
\text { 3- Not very clear } \\
\text { 4- Not clear at all }\end{array}$ & 90.75 & 89.7 \\
\hline K3 & $\begin{array}{l}\text { Q3: How difficult is it to handle all } \\
\text { the practical work's elements? }\end{array}$ & $\begin{array}{l}\text { 1-Very difficult } \\
\text { 2- Quite difficult } \\
\text { 3- minor difficul- } \\
\text { ties } \\
\text { 4- No problem }\end{array}$ & 90.15 & 80.2 \\
\hline K4 & $\begin{array}{l}\text { Q4: According to you, does this } \\
\text { practical work match the theoretical } \\
\text { concepts? }\end{array}$ & $\begin{array}{l}\text { 1-Yes, totally } \\
\text { 2- Yes, partially } \\
\text { 3- Not so much } \\
\text { 4- Not at all }\end{array}$ & 95.5 & 89.1 \\
\hline \multicolumn{5}{|c|}{ Open questions } \\
\hline & Question & $\begin{array}{c}\text { Time Hands-on } \\
\text { Pw }\end{array}$ & \multicolumn{2}{|c|}{ Time Remote PW } \\
\hline $\begin{array}{l}\text { Q5 } \\
\text { tic }\end{array}$ & $\begin{array}{l}\text { : How long did you take for this prac- } \\
\text { l work (Min)? }\end{array}$ & $60 \mathrm{~min}$ & \multicolumn{2}{|c|}{$40 \mathrm{~min}$} \\
\hline Q6 & $\begin{array}{l}\text { : How long did you take to process } \\
\text { results and write this practical work } \\
\text { ort (Min)? }\end{array}$ & $104 \min$ & \multicolumn{2}{|c|}{$75 \min$} \\
\hline
\end{tabular}

Analysis of the responses in Table 1 shows some important points. According to the results of the closed questions, the majority of the students' answers are favorable, varying between the first and second choice answers for each question in the two practical work systems. The results of the K-factor in the practical laboratory are always higher than the remote laboratory factor, which highlights two conclusions: the first is that the hands-on laboratory is the most preferred by the students, the second is that the minor difference between the value of the K-factor in the hands-on and the remote laboratory proves that the IoT system has succeeded in transmitting the same knowledge at a distance as well as it can be adapted perfectly in unexpected eventual cases such as the case of covid'19 to save the practical learning of the students. Based on the two openended questions presented in Table 1, it is clear that practical distance work can save students' time.

\section{Conclusion}

In this paper, we have outlined the motivations that have led to meeting the needs of new practical teaching methods in open access university in order to save time, space, and money without loss of quality or quantity in practical teaching. Indeed, in this work, we have proposed the conception and implementation of an IoT system allowing the 
remote realization of several analog electronics laboratories. This solution is mainly based on the Red Pitaya STEMLab card, especially for communication and remote control by the RIP protocol, for measurements and data acquisition, to which is added an electronic card allowing an intelligent selection between the different integrated practical works and other manipulations carried out externally as extensions according to the needs of the teacher by an analog multiplexing technique that allows the routing of input/output signals to and from the selected manipulations.

The proposed board incorporates two digital potentiometers that are controlled via the SPI protocol to continuously monitor the resistance value, the first one is connected to the six manipulations integrated on the board, and the second one is dedicated to the modification of the resistance value in the extensions created by the teacher outside the board, this property is an added value to the quality of the board.

We have presented the various components of this system, namely the chosen architecture, the developed board for practical work, the used software to develop the enduser interface, and a $\mathrm{Pw}$ prototype to compare our remote system with the traditional method of practical work in open access universities. The Rc charging and discharging circuit was considered as a prototype of study in this work, the results show that perceptions of students toward the remote IoT system are very similar to those of handson. This promising first experience has proven that it is able of competing with the traditional system, also it can be a complementary alternative to the current learning system.

We are treating as future work the development of a more complete platform using the python RIP protocol and EJSS and to study the issues of accessibility and load increase, and we are looking forward to creating a window of conversation with the supervisor in order to accompany the experiments.

\section{$7 \quad$ Acknowledgement}

The authors wish to thank the Computer Science and Automation Department of the National University of Distance Education, Madrid, Spain, for the help they have provided in carrying out this work, in particular on the RIP communication protocol which they have developed and made available to the public.

\section{References}

[1] C. Choi Chea, J. Tan JuatHuan, "Higher education 4.0: the possibilities and challenges", Journal of Social Sciences and Humanities, Vol. 5, No. 2, pp. 81-85, 2019

[2] D. Schugurensky. C. Wolhuter. "Teacher's Education and Global Citizenship Education," In book: Global Citizenship Education and Teacher Education, pp.1-19. 2020 https://doi. org/10.4324/9781351129848-1

[3] G. Van Dijik. A. Meiji, E.Savelsbergh. "Maker Education: Opportunities and Threats for Engineering and Technology Education". Education: Building A Sound Mind and Sound Body, Publisher: A Publication of the Faculty of Education, Lagos State University, Ojo, Lagos State, pp.101 - 1-5. 2020. 
[4] A. Moulay taj, A. Abouhilal, N. Taifi, J.Antari, A. Malaoui "Low-cost smart architecture for classroom response system using raspberry: raspcrs", International Journal of Engineering \& Technology, 7 (4.32) 79-83, 2018

[5] J. Wellington and G. Ireson "Practical Work in Science education". Science Learning, Science Teaching, pp.166-177, September 2017. https://doi.org/10.4324/9781315623429-7

[6] A. Malaoui, M. Kherallah, L. Ghomri, M. Raoufi, G. Andrieu, T. Fredon, and D. Barataud. "Implementation and validation of a new strategy of online practical works of power electronics for embedded systems," Int. J. Online Eng., vol. 13, no. 4, pp. 29-44. ,2017. https://doi.org/10.1007/978-3-319-60834-1_16

[7] E. Fabregas, G. Farias, S. Dormido-Canto, S Dormido, and F. Esquembre, "Developing a remote laboratory for engineering education," Comput. Educ., vol. 57, no. 2, pp. 1686-1697, 2011. https://doi.org/10.1016/j.compedu.2011.02.015

[8] Antonian, L. (2015). "Armenia: Higher Education Problems and Perspectives". International Higher Education, (19). https://doi.org/10.6017/ihe.2000.19.6862

[9] K. Narasimhamurthy, K. Thanmayi, T. Bhuvana, H. Chaitra, k. Spurthy, T. Kashyap. "Analysis of Filter Circuits in Remote Lab" Cyber-physical Systems and Digital Twins, pp.612622, January 2020. https://doi.org/10.1007/978-3-030-23162-0_55

[10] L. Pellegrino, C. Sandroni, E. Bionda, D. Pala, D. Lagos, N. Hatziargyriou. Akroud, N "Remote Laboratory Testing Demonstration" in Energies 13(9):2283.May 2020. https://doi. org/10.3390/en13092283

[11] I. Titov, A. Glotov, I. Vlasov, J. Mikolnikov. "Labicom Labs 2015: Remote Laser Virtual and Remote Lab, Global Navigation Satellite Systems Virtual and Remote Lab, Microwave Amplifier Remote Lab" International Journal of Online Engineering (iJOE), 12(04):17, April 2016. https://doi.org/10.3991/ijoe.v12i04.5076

[12] M. Guinaldo, L. de la Torre, R. Heradio, and S. Dormido, "A virtual and remote control laboratory in moodle: The ball and beam system,"in10th IFAC Symposium on Advances in Control Education, 2013. https://doi.org/10.3182/20130828-3-uk-2039.00021

[13] Ruben Heradio, J. Chacón, H.Vargas, J. Sanez, L. de la Torre, and S. Dormido, "OpenSource Hardware in Education: a Systematic Mapping Study", IEEE Access Journal, November 2018, https://doi.org/10.1109/access.2018.2881929

[14] F. Amiel, D. Abboud, and M. Trocan, "A Project Oriented Learning Experience for Teaching Electronics Fundamentals,” IEEE Commun. Mag., pp. 98-100, Dec. 2014 https://doi. org/10.1109/mcom.2014.6979959

[15] I. Ugarte, V. Fernández and P. Sánchez, "Motivation of students in the learning of digital electronics through the double integration: Remote/presential work and theoretical/laboratory classes," presented at the Technologies Applied to Electronics Teaching (TAEE), Vigo, Spain, 2012. https://doi.org/10.1109/taee.2012.6235408

[16] J. Mendoza, J. Villadangos Carrizo and F. Rodríguez Sánchez, "Project based learning experiences for embedded systems design," in Technologies Applied to Electronics Teaching (TAEE), Sevilha, Spain, 22-24 June 2016. https://doi.org/10.1109/taee.2016.7528370

[17] E. Ortiz-Rivera and M. Castro-Sitiriche, "Work in progress - integration of hands-on-laboratory experience of power electronics and renewable energy applications," in 39th IEEE Frontiers in Education Conference, Oct 2009, pp. 1-2. https://doi.org/10.1109/fie.2009. $\underline{5350814}$

[18] A. Bousbaine and M. Eljarh, "A practical project approach for teaching experimental power electronics," in 46th International Universities' Power Engineering Conference (UPEC), Sept 2011, pp. 1-6. 
[19] J. Hudgins, A. Monti, and R. Dougal, "Control system laboratory: a power electronics teaching experience," in Proc. IEEE Workshop on Computers in Power Electronics, June 2002, pp. 112-116. https://doi.org/10.1109/cipe.2002.1196724

[20] H. Shen et al., "Conducting laboratory experiments over the Internet," IEEE Trans. Educ., vol. 42, no. 3, pp. 180-185, Aug. 1999.

[21] B. Kollöffel and T. De Jong, "Conceptual understanding of electrical circuits in secondary vocational engineering education: Combining traditional instruction with inquiry learning in a virtual lab," J. Eng. Educ., vol. 102, no. 3, pp. 375-393, 2013 https://doi.org/10.1002/ jee. 20022

[22] U. Hernández-Jayo and J. García-Zubía, "Remote measurement and instrumentation laboratory for training in real analog electronic experiments," Measurement, vol. 82, pp. 123134, Mar. 2016. https://doi.org/10.1016/j.measurement.2015.12.017

[23] M. A. Marques et al., "How remote labs impact on course outcomes: Various practices using VISIR,” IEEE Trans. Educ., vol. 57, no. 3, pp. 151-159, Aug. 2014. https://doi.org/10. $\underline{1109 / \text { te. } 2013.2284156}$

[24] J. Garcia-Zubia, U. Hernandez-Jayo, M. Guenaga and I. Gustavsson "Empirical analysis of the use of the VISIR remote lab in teaching analog electronics," IEEE Trans. Educ., vol. 60, no. 2, May. 2017. https://doi.org/10.1109/te.2016.2608790

[25] H. Considine, M.Teng, A.Nafalski, Z. Nedic. " Recent developments in remote laboratory Netlab", Global Journal of Engineering Education, vol 18, N 1 January 2016. https://doi.org/ $\underline{10.1109 / \text { educon} 45650.2020 .9125230}$

[26] A. Bagnasco, M. Chirico, A.M. Scapolla, "A New and Open Model to Share Laboratories on the Internet", IEEE Transaction on Instrumentation and Measurement, June 2005, ISSN 0018-9456, Volume 54, pp 1111-1117. https://doi.org/10.1109/tim.2005.847205

[27] A. V. Fidalgo et al., "Adapting remote labs to learning scenarios: Case studies using VISIR and RemotElectlab," IEEE Revistalberoamericana de Tecnología del Aprendizaje, vol. 9, no. 1, pp. 33-39, Feb. 2014 https://doi.org/10.1109/rita.2014.2302071

[28] https://github.com/UNEDLab/rip-spec

[29] https://www.redpitaya.com/

[30] https://www.um.es/fem/EjsWiki/

\section{Authors}

Amine Moulay Taj received his M.Sc. degree in Computer Science from the Sultan Moulay Slimane University of Beni Mellal, Morroco, in 2016. He is currently pursuing a Ph.D. degree with the Research Team in Electronics, Instrumentation and Measurement at the Polydisciplinaire University of Beni Mellal, and he is also a visiting researcher at UNED Madrid. His interests include the integration of information and communication technologies to improve the quality of practical teaching, control engineering, virtual and remote laboratories.

Jesus Chacon Sombria received a degree in Automation and Industrial Electronics Engineering from University of Cordoba, Spain, in 2010, and the Ph.D. degree in Computer Science from UNED,Madrid, Spain, in 2014. He was as researcher at UNED from 2014 to 2018 and currently an assistant professor at Department of Computer Architecture and Automation Universidad Complutense de Madrid, 28040.Madrid, Spain, since 2019. His current research interests include event-based control systems and remote and virtual labs in control engineering. jeschaco@ucm.es 
Ahmed Gaga received his Engineering degree in Electronics and Embedded Systems from the University of sciences and technologies, Fez, Morocco in 2013, he was a research professor at UPF University in Fez between 2015 and 2018. Currently, he is an Assistant Professor at USMS University, Beni Mellal, Morocco. He works in Electrical Engineering, especially renewable energies and intelligent systems. His research interest is Electronics; Microcontroller based embedded systems, Photovoltaic applications and Control theory Gaga.ahmad@gmail.com

Abdelmoula Abouhilal is a Ph.D. student with the research team in Electronic, Instrumentation and Measurement in the polydisciplinary faculty of Beni Mellal. His research interests include remote laboratories, instrumentation and embedded systems. A.abouhilal@usms.ma

Abdessamad Malaoui is an Associate Professor in the Physics department of the Polydisciplinary faculty of Sultan Moulay Slimane University, Béni Mellal, Morocco. His research interests are in electronics, Industrial computer, $\mu \mathrm{P} \& \mu \mathrm{C}$, renewable energy, PV system performance monitoring. A.Malaoui@usms.ma

Article submitted 2020-11-09. Resubmitted 2020-12-12. Final acceptance 2020-12-13. Final version published as submitted by the authors. 